\title{
Recombination zone in mixed-host organic light-emitting devices
}

\author{
Chih-Hung Hsiao, Yan-Hau Chen, Tien-Chun Lin, Chia-Chiang Hsiao, and Jiun-Haw Lee ${ }^{a}$ \\ Graduate Institute of Electro-Optical Engineering, National Taiwan University, No. 1, Sec. 4, \\ Roosevelt Road, Taipei, Taiwan 106, Republic of China and Department of Electrical Engineering, \\ National Taiwan University, No. 1, Sec. 4, Roosevelt Road, Taipei, Taiwan 106, Republic of China
}

(Received 13 May 2006; accepted 31 August 2006; published online 19 October 2006)

\begin{abstract}
In this letter, the authors had quantitatively investigated the recombination zone in the mixed-host (MH) emitting layer (EML) of an organic light-emitting device with different mixed ratios experimentally and theoretically. The MH-EML consisted of a hole-transport layer (HTL) and an electron-transport layer fabricated by coevaporation. When the mixed ratio of the HTL in the EML increases, the driving voltage increases then decreases; this can be well demonstrated by an electrical model with different carrier mobilities. A blueshift was also observed due to the solid state solvation effect combined with the exciton shift from the anode to the cathode side. () 2006 American Institute of Physics. [DOI: 10.1063/1.2361266]
\end{abstract}

In 1987, Tang and Vanslyke demonstrated organic lightemitting devices (OLEDs), consisting of several layers of amorphous organic films with the thicknesses of tens of nanometers, with a driving voltage of less than $10 \mathrm{~V} .{ }^{1}$ From then on, OLEDs have attracted much attention because of their advantages such as low-power consumption, high brightness, high contrast, and potentially low cost. ${ }^{2}$ However, their operation lifetime is still one of the major limitations for OLED applications as compared to other competitive display technologies such as semiconductor lightemitting diodes and liquid crystal displays. ${ }^{3}$

In order to elongate the operation lifetime, a mixed-host (MH) structure had been introduced as the host of the emitting layer (EML). ${ }^{4,5}$ In a conventional heterojunction (HJ) OLED, the interface of the hole-transporting layer (HTL) and EML builds up a barrier to enhance carrier confinement, which improves the device efficiency. ${ }^{1}$ However, the accumulated holes in the EML near the interface formed chemically unstable cations, which accelerated the formation of nonradiative trapping centers, thus resulting in luminance decay and voltage increase of an OLED. ${ }^{6}$ By coevaporating the HTL and the electron-transport layer (ETL) material as the host of the EML, the HTL/EML interface became blurry. This reduced carrier accumulation can broaden the emission zone, elongating the operation lifetime. This blurring boundary raises the probability of penetration of holes into EML so that excitons are generated over wider region in EML instead of narrower region around the interface of HTL/EML. In this regard, the broadening of emission zone, which indicates that the leakage of the carriers to the electrodes is dominant, decreases device efficiency. ${ }^{7}$ Typically, the operation lifetime of MH-OLEDs can be extended by a factor of 6 as compared to the conventional HJ ones. 8

However, in the absence of the carrier confinement of the conventional HJ structure, the MH-OLED exhibits a higher carrier leakage and lower current efficiency. Furthermore, the bipolar characteristic of the EML would shift the recombination zone from the interface into the EML, causing a reduction in current efficiency due to the quenching by the metal electrodes. To optimize the device characteristics of a MH-OLED, different layer thicknesses and mixed ratios

\footnotetext{
${ }^{a)}$ Electronic mail: jhlee@cc.ee.ntu.edu.tw
}

were tested and results were discussed qualitatively. ${ }^{10}$ In our experiment we had developed an electrical and an optical model from basic equations to quantitatively simulate the current density-voltage $(J-V)$ curves and electroluminescence (EL) spectra of the MH-OLEDs with different mixed ratios. Electron and hole mobilities of the $\mathrm{MH}$ layers with different mixture concentrations were extracted, which can well explain the $J-V$ characteristics of MH-OLEDs. Based on the mobility values, carrier and exciton distributions can be used to calculate the EL spectral shift.

Our fabrication and measurement details are shown in Ref. 11. ${ }^{11}$ Here, we used $N, N^{\prime}$-diphenyl$N, N^{\prime}$-bis(1-napthyl)-1,1'-biphenyl-4,4'-diamine (NPB) as the HTL material and tris-(8-hydroxyquinoline) aluminum $\left(\mathrm{Alq}_{3}\right)$ as the ETL material. The EML was a mixture of NPB and $\mathrm{Alq}_{3}$ with different mixed ratios. LiF and aluminum (Al) were served as the electron-injection layer and the cathode. Our device structures were ITO/NPB $(20 \mathrm{~nm}) /$ NPB : $\mathrm{Alq}_{3}(80 \mathrm{~nm}) / \mathrm{Alq}_{3}(20 \mathrm{~nm}) / \mathrm{LiF}(1.2 \mathrm{~nm}) / \mathrm{Al}(150 \mathrm{~nm})$. Devices A, B, C, D, and E corresponded to the NPB: $\mathrm{Alq}_{3}$ mixed ratios of $0: 1,1: 3,1: 1,3: 1$, and 1:0, respectively. In our simulations, we developed a numerical method for investigating the electrical properties of an OLED based on the drift-diffusion equations containing charge carrier drift with field-dependent mobility, thermionic emission, HJ interface, and recombination process. ${ }^{12,13}$ The input data of such an electrical simulation included the carrier mobility and band diagram of the organic materials, and the layer thicknesses of an OLED. From this electrical simulation model, we achieved the $J-V$ characteristics and the distribution of the electric field, carrier and exciton concentrations, and recombination rate in an OLED. The exciton distribution was used as the input of the optical simulation program based on general electromagnetic theory. ${ }^{14}$ In addition to the exciton distribution, the other input parameters comprised the layer thickness, the real and imaginary parts of refractive index of each layer, and the photoluminescence (PL) of the emitter. The outputs of this program encompassed the spectrum, intensity, and viewing angle of an OLED.

Figure 1(a) shows the experimental and simulated $J-V$ characteristics. The solid lines and the dots show the simulation and experiment results, respectively. The values of the highest occupied molecule orbital (HOMO) and the lowest 

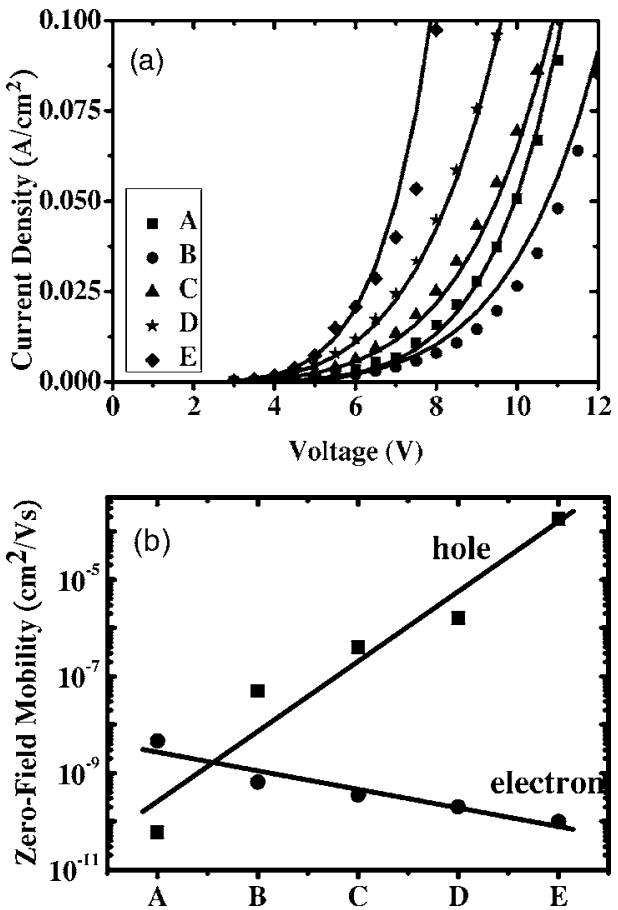

FIG. 1. (a) Experimental (dots) and stimulated (solid lines) $J$ - $V$ characteristics of the five devices. (b) Fitted zero-field carrier mobilities of EML with different mixed ratios.

unoccupied molecule orbital (LUMO) of NPB $\left(\mathrm{Alq}_{3}\right)$ are $5.4 \mathrm{eV}(3.0 \mathrm{eV})$ and $2.4 \mathrm{eV}(5.7 \mathrm{eV})$. The work functions of indium tin oxide (ITO) and LiF:Al are 4.9 and $3.2 \mathrm{eV} .{ }^{15} \mathrm{In}$ our simulation, the only fitting parameter was electric-field $(F)$ dependent carrier mobility $\mu$ of the MH layers, following the Poole-Frenkel form: ${ }^{16}$

$$
\mu=\mu_{0} \exp \left(\sqrt{\frac{F}{E_{0}}}\right),
$$

where $\mu_{0}$ is the zero-field mobility and $E_{0}$ is a constant. In our fitting, the $\mu_{o}$ and $E_{o}$ values of NPB for holes were $\mu_{p o}=1.8 \times 10^{-4} \mathrm{~cm}^{2} / \mathrm{V} \mathrm{s}$ and $E_{p o}=2 \times 10^{5} \mathrm{~V} / \mathrm{cm}$, and for electrons were $\mu_{n o}=1 \times 10^{-10} \mathrm{~cm}^{2} / \mathrm{V} \mathrm{s}$ and $E_{n o}=1$ $\times 10^{8} \mathrm{~V} / \mathrm{cm}$. The $\mu_{o}$ and $E_{o}$ values of $\mathrm{Alq}_{3}$ for holes were $\mu_{p o}=6 \times 10^{-11} \mathrm{~cm}^{2} / \mathrm{V} \mathrm{s}$ and $E_{p o}=1.25 \times 10^{4} \mathrm{~V} / \mathrm{cm}$, and for electrons were $\mu_{n o}=4.6 \times 10^{-9} \mathrm{~cm}^{2} / \mathrm{V} \mathrm{s}$ and $E_{n o}=1.4$ $\times 10^{4} \mathrm{~V} / \mathrm{cm}$, respectively. ${ }^{17,18}$ In terms of the mobilities in $\mathrm{MH}$ layer, the $\mu_{o}$ values were varied to fit the experimental results on condition of the constant $E_{o}$ values. As shown in Fig. 1(b), the fitted hole (electron) mobility increases (decreases) almost linearly with increasing (decreasing) HTL material composition in mixed ratio.

The holes and electrons mainly hop through the NPB and $\mathrm{Alq}_{3}$ molecules, respectively. In light of the energetically favorite, the hole and electron mobilities in $\mathrm{MH}$ layers were lower than the values in the pure NPB and $\mathrm{Alq}_{3}$. On the other hand, as shown in Fig. 1(a), the driving voltage increases and then decreases with increasing HTL material concentration. In the case of conventional HJ device, the thicker NPB exhibited lower driving voltage with the constant total organic thickness since the hole mobility of NPB was two orders of magnitude higher than the electron mobility of $\mathrm{Alq}_{3}{ }^{15}$ In $\mathrm{MH}$ devices, the monotonically decreasing driving voltage did not occur as expected in HJ devices. The discrepancy is the bipolar carrier transport property in $\mathrm{MH}$ device. Raising Downloaded 06 Feb 2009 to 140.112 .113 .225 . Redistribution subje

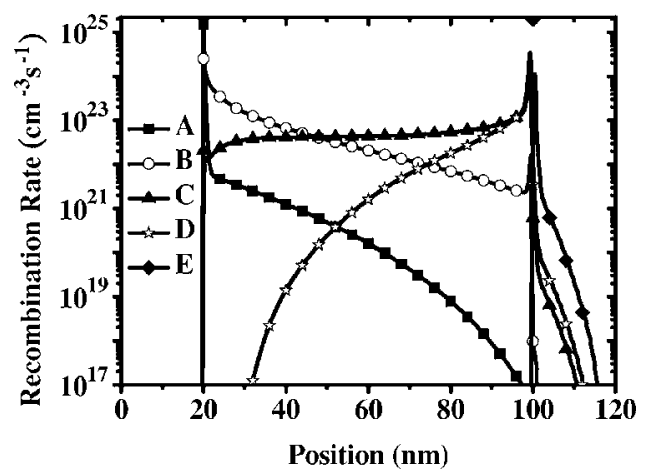

FIG. 2. Recombination distribution in MH-OLEDs. 20 and $100 \mathrm{~nm}$ indicate the interface of $\mathrm{NPB} / \mathrm{MH}$ and $\mathrm{MH} / \mathrm{Alq}_{3}$, respectively.

NPB concentration in MH layer led to the enhanced hole current and reduced electron current. The hole-current enhancement did not compensate the electron-current decline when the HTL ratio increased from 0\% to 25\% (from devices A to B). This unbalanced change in opposite carrier currents explains the voltage increasing in device $\mathrm{B}$ as compared with device A. The sequential increase of HTL material concentration from $25 \%$ to $100 \%$ resulted in the voltage reduction from devices $\mathrm{C}$ to $\mathrm{E}$ on account of rapidly boost of hole current.

Figure 2 shows the simulation results of the recombination rate distribution in the MH-OLEDs. With increased NPB ratio in $\mathrm{MH}$ layer, the recombination zone shifted from the HTL/EML interface (device A), broadenened (devices B, C, and D), and finally shrank to the interface of EML/ETL (device E). Since the energetic offset of the LUMO between NPB and $\mathrm{Alq}_{3}$ was larger than that of HOMO, the penetration of holes into the $\mathrm{Alq}_{3}$ layer (devices $\mathrm{C}, \mathrm{D}$, and $\mathrm{E}$ ) was easier than that of electrons into the NPB (devices A and B). The wide recombination zone of the MH-OLED is well known to interpret its longer lifetime as compared with that of the HJ-OLEDs. ${ }^{7}$

We have quantitatively demonstrated the recombination zone distribution and the mobility change with different mixed ratios. Once the recombination distribution was obtained, we used it to derive the exciton distribution, an input of our optical simulation model as described in Ref. $19 .{ }^{19}$ The other input parameters included refractive index $(n)$ and extinction coefficient $(k)$ values of each layer, and PL spectra of the EML. ${ }^{20}$ Figure 3 shows the $n-k$ values at different wavelengths for the MH thin films. We can see that the refractive index of pure NPB was the highest. As the $\mathrm{Alq}_{3}$ ratio in $\mathrm{MH}$ layer increased, the refractive index values decreased monotonically. The extinction coefficients of $\mathrm{NPB}, \mathrm{Alq}_{3}$, and the MH-EML were negligibly nearly zero within the emission spectrum (i.e., $450-700 \mathrm{~nm}$ ). Furthermore, the $n$ - $k$ values of the MH layers were close to the linear interpolations of pure NPB and $\mathrm{Alq}_{3}$. This means that $\mathrm{MH}$ layers were physically mixed without chemical interaction from mesoscopic viewpoint.

It was a typical solid state solvation effect (SSSE) that the PL peak exhibited a blueshift from 526, 525, 521 to $517 \mathrm{~nm}$ when the NPB ratio changed from $0 \%, 25 \%$, $50 \%$ to $75 \%{ }^{21}$ Figure 4 shows the measured EL spectra of all devices. The measured and simulated spectral peaks blueshifted from 555, 543, 537, 529 to $522 \mathrm{~nm}$ and 557, 546, 537,535 to $531 \mathrm{~nm}$, for devices A, B, C, D, and E, respecto AlP license or copyright; see http://apl.aip.org/apl/copyright.jsp 

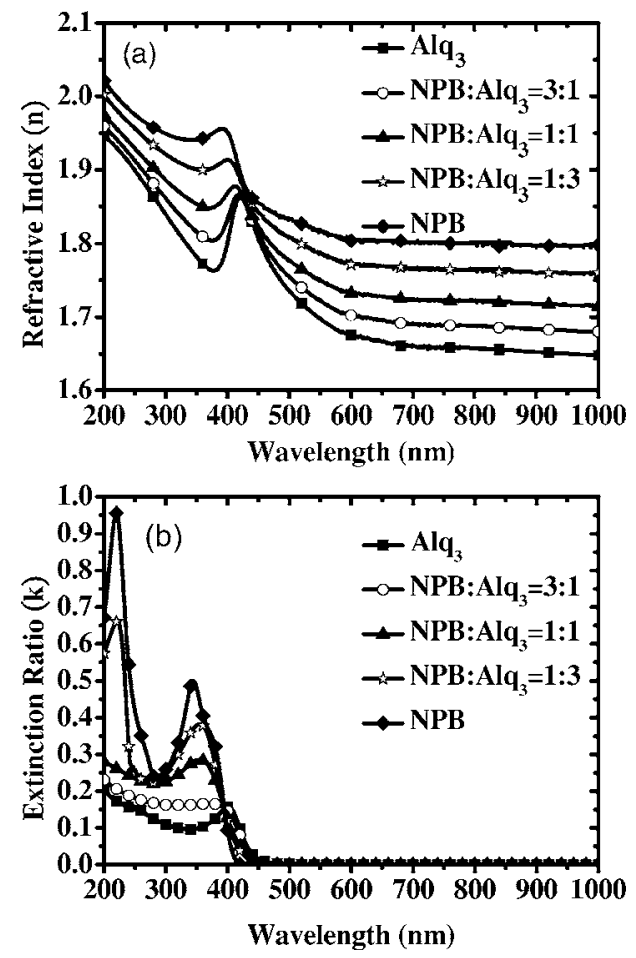

FIG. 3. (a) Refractive index and (b) extinction coefficient spectra of the MH-EMLs.

tively. The experimental results matched well with that from the simulation program. Note that there was no adjustable input parameter in this simulation. The peak shift of the EL was much more than that of the PL, indicating the blueshift with increased NPB ratio came not only from the SSSE but also from the optical interference effect. Typically, in the studied range, the EL spectrum reveals a blueshift when the exciton distribution shifts from the anode to the cathode side, ${ }^{13}$ as is shown in Fig. 2.

In conclusion, the recombination distributions in the MH-EML with different mixed ratios were studied. We found that the recombination zone that initially resided at the HTL/EML interface extended into the MH layer and piled up at the EML/ETL interface as the increasing HTL material ratio in $\mathrm{MH}$ layer. A quantitatively electrical model, based on the mobility change with different mixing ratios, was established. Good agreement between experimental and simulated EL spectra suggests that the EL spectral shift came not only from the intrinsic PL spectral shift but also from the extrinsic optical interference effect that originated from the distribution of radiative dipoles, namely, exciton distribution.

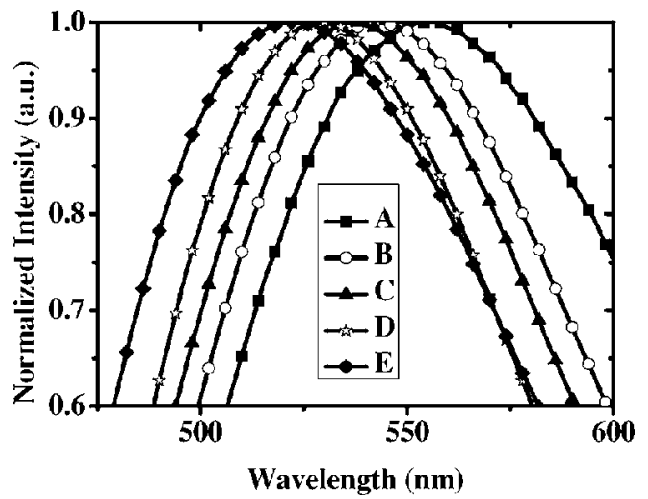

FIG. 4. Measured EL spectra of the five devices.

This work was supported by the National Science Council, R.O.C., under Grant No. NSC 95-2221-E-002-305 and by Aixtron Corporation.

${ }^{1}$ C. W. Tang and S. A. VanSlyke, Appl. Phys. Lett. 51, 913 (1987).

${ }^{2}$ C. W. Tang and S. A. VanSlyke, J. Appl. Phys. 65, 3610 (1989).

${ }^{3}$ J. H. Lee, J. J. Huang, C. C. Liao, P. J. Hu, and Y. Chang, Chem. Phys. Lett. 402, 335 (2005).

${ }^{4}$ H. Aziz, Z. D. Popovic, N.-X. Hu, A.-M. Hor, and G. Xu, Science 283, 1900 (1999).

${ }^{5}$ A. B. Chwang, R. C. Kwong, and J. J. Brown, Appl. Phys. Lett. 80, 725 (2002).

${ }^{6}$ D. Y. Kondakov, J. R. Sandifer, C. W. Tang, and R. H. Young, J. Appl. Phys. 93, 1108 (2003).

${ }^{7}$ V. E. Choong, S. Shi, J. Curless, C. L. Shieh, H. C. Lee, J. Shen, and J. Yang, Appl. Phys. Lett. 75, 172 (1999)

${ }^{8}$ J. Kalinowski, L. C. Palilis, W. H. Kim, and Z. H. Kafafi, J. Appl. Phys. 94, 7764 (2003).

${ }^{9}$ S. W. Liu, C. A. Huang, J. H. Lee, K. H. Yang, C. C. Chen, and Y. Chang, Thin Solid Films 453-454, 312 (2004).

${ }^{10}$ J. H. Lee, C. I. Wu, S. W. Liu, C. A. Huang, and Y. Chang, Appl. Phys. Lett. 86, 103506 (2005)

${ }^{11}$ J. H. Lee, M. H. Wu, C. C. Chao, H. L. Chen, and M. K. Leung, Chem. Phys. Lett. 416, 234 (2005).

${ }^{12}$ P. S. Davids, I. H. Campbell, and D. L. Smith, J. Appl. Phys. 82, 6319 (1997).

${ }^{13}$ E. Tutis, M. N. Bussac, B. Masenelli, M. Carrard, and L. Zuppiroli, J. Appl. Phys. 89, 430 (2001).

${ }^{14}$ H. C. Chen, J. H. Lee, C. C. Shiau, C. C. Yang, and Y. W. Kiang, J. Lightwave Technol. 24, 2450 (2006).

${ }^{15}$ S. J. Martin, G. L. B. Verschoor, M. A. Webster, and A. B. Walker, Org. Electron. 3, 129 (2002).

${ }^{16}$ W. Brütting, S. Berleb, and A. G. Mückl, Org. Electron. 2, 1 (2001).

${ }^{17}$ B. J. Chen, W. Y. Lai, Z. Q. Guo, C. S. Lee, S. T. Lee, and W. A. Gambling, Appl. Phys. Lett. 75, 4010 (1999).

${ }^{18}$ B. Chen, C. S. Lee, S. T. Lee, P. Webb, Y. C. Chan, W. Gambling, H. Tian, and W. Zhu, J. Appl. Phys. 39, 1190 (2000).

${ }^{19}$ B. W. D. Andrade, M. E. Thompson, and S. R. Forrest, Adv. Mater. (Weinheim, Ger.) 14, 147 (2002).

${ }^{20}$ J. H. Lee, C. C. Liao, P. J. Hu, and Y. Chang, Synth. Met. 144, 279 (2004).

${ }^{21}$ V. Bulović, R. Deshpande, M. E. Thompson, and S. R. Forrest, Chem. Phys. Lett. 308, 317 (1999). 